\title{
Identification of Metal-Binding Peptides and Their Conjugation onto Nanoparticles of Superparamagnetic Iron Oxides and Liposomes
}

Tomonobu Kodama $t^{1,2}$, Akifumi Yoshihara $\ddagger^{1}$, Isha Goel ${ }^{1}$, Masaki Sekino ${ }^{1,3}$, Akihiro Kuwahata $^{3}$, Atsushi Yoshimori ${ }^{4}$, Yuichi Murayama ${ }^{2}$, Kazuhiko Ishihara ${ }^{5}$, Kristina N Ekdahl $^{6,7}$, Bo Nilsson ${ }^{6}$, and Yuji Teramura*1,6

$¥$ Contributed equally

*Corresponding Author

Yuji TERAMURA

Tel: +81(0)3-5841-1174

E-mail: teramura@,bioeng.t.u-tokyo.ac.jp

${ }^{1}$ Department of Bioengineering, School of Engineering, The University of Tokyo, 73-1 Hongo, Bunkyo-ku, Tokyo, 113-8656, Japan

${ }^{2}$ Department of Neurosurgery, The Jikei University School of Medicine, Tokyo, 1058461, Japan.

${ }^{3}$ Department of Electrical Engineering and Information Systems, School of Engineering, The University of Tokyo, Tokyo, 113-8656, Japan.

${ }^{4}$ Institute for Theoretical Medicine, Inc., 26-1 Muraoka-Higashi 2-chome, Fujisawa, Kanagawa 251-0012, Japan

${ }^{5}$ Department of Material Engineering, School of Engineering, The University of Tokyo, 7-3-1 Hongo, Bunkyo-ku, Tokyo, 113-8656, Japan

${ }^{6}$ Department of Immunology, Genetics and Pathology (IGP), Uppsala University, Dag Hammarskjölds väg 20, SE-751 85, Uppsala, Sweden

${ }^{7}$ Linnaeus Center of Biomaterials Chemistry, Linnaeus University, SE-391 82 Kalmar, Sweden 


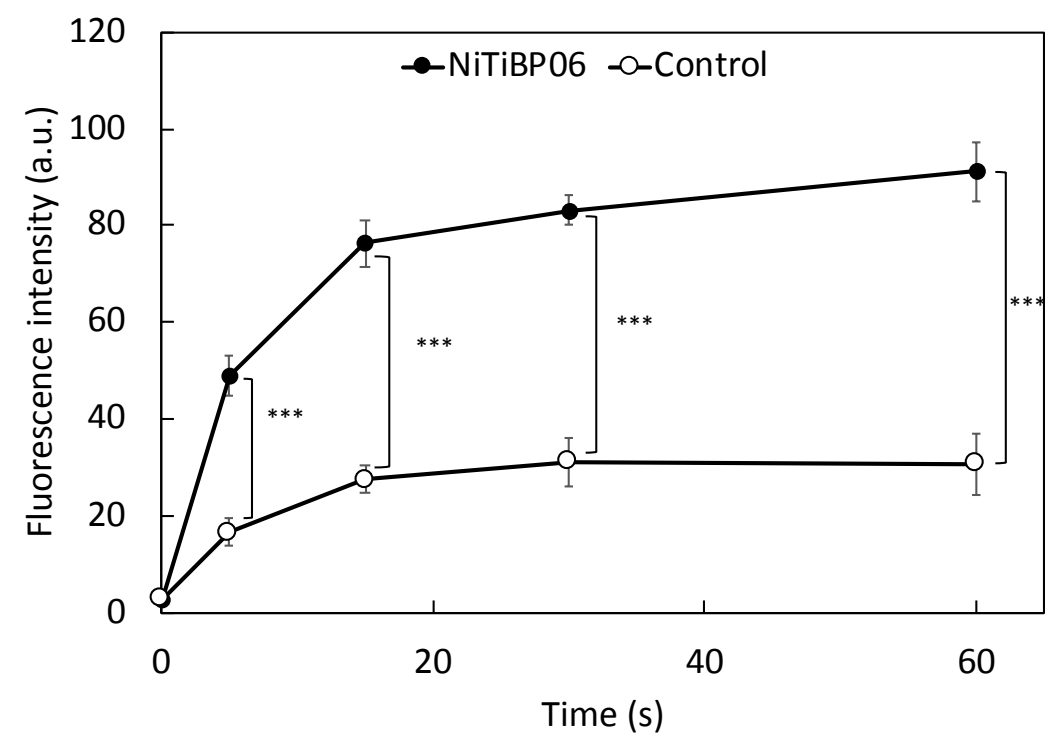

Supplementary Figure S1. Binding behavior of Ni-Ti-binding peptide with time. After Ni-Ti stent was exposed to human plasma, the stent was incubated in FITClabelled NiTiBP06 solution $(100 \mu \mathrm{g} / \mathrm{mL})$ and the fluorescence from the surface was measured over time by upright fluorescence microscope. As a control, FITCCoCrBP02 was used. Error bars indicate standard deviation; $n=3$.
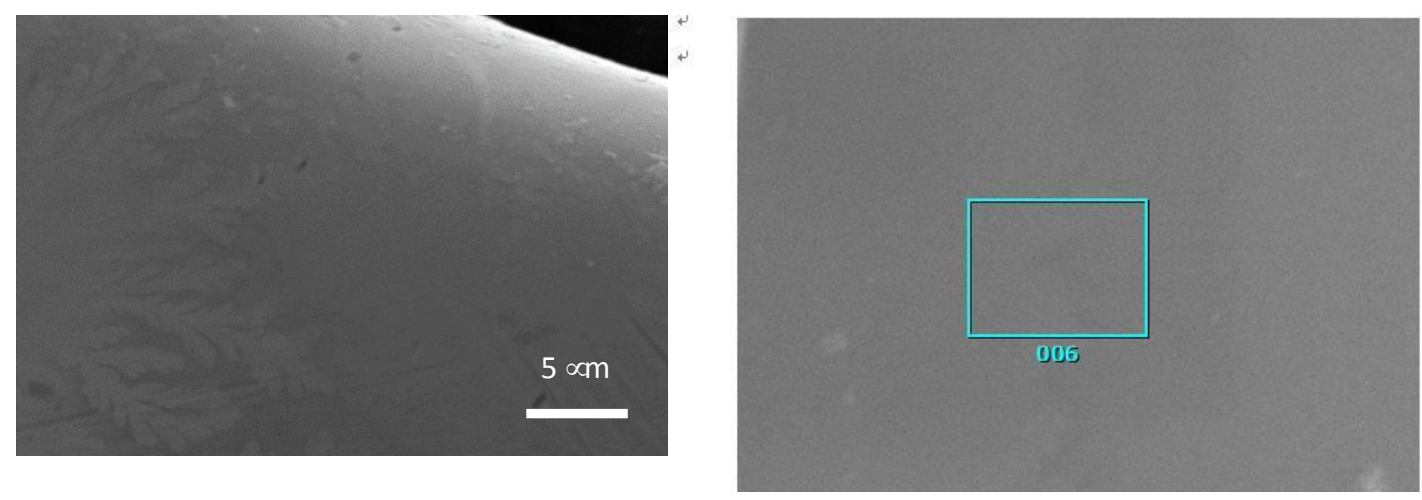

Supplementary Figure S2. SEM image of Ni-Ti stents after treatment with SPIOs and the elemental analysis. 

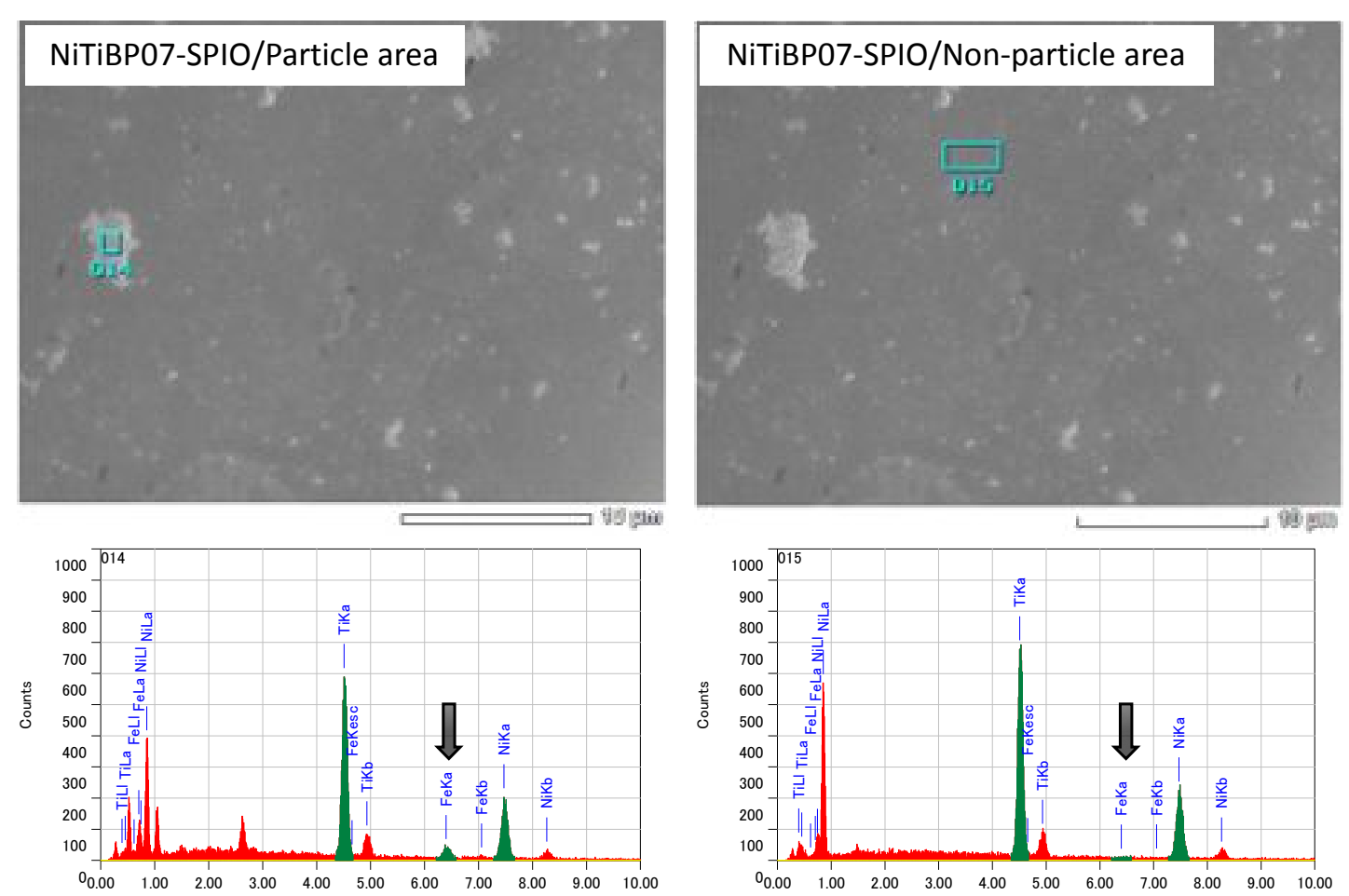

\begin{tabular}{l|r|r} 
Element & Weight (\%) & Atoms (\%) \\
\hline Ti K & 42.53 & 47.44 \\
\hline Fe K & 5.57 & 5.33 \\
\hline Ni K & 51.90 & 47.23 \\
\hline \hline Total & 100.00 & 100.00
\end{tabular}

\begin{tabular}{l|r|r} 
Element & Weight (\%) & Atoms (\%) \\
\hline Ti K & 45.33 & 50.40 \\
\hline Fe K & 0.17 & 0.17 \\
\hline Ni K & 54.49 & 49.43 \\
\hline \hline Total & 100.00 & 100.00
\end{tabular}

Supplementary Figure S3. Elemental analysis from SEM images of Ni-Ti stents after treatment with peptide-conjugated SPIOs. The area where the particles were observed (left) and not observed (right) were analyzed. Arrows indicate detected Fe elements. 